\title{
Correction to: Hepatotoxicity associated with PD-1 blockade antibodies in cancer patients co-infected with hepatitis B virus
}

\author{
Zuan Lin ${ }^{1} \cdot$ Xuanye Zhang ${ }^{2} \cdot$ Yixin Zhou ${ }^{3} \cdot$ Chen Chen ${ }^{4} \cdot \mathrm{Li}^{-n a ~ \mathrm{He}^{2}} \cdot$ Haifeng $\mathrm{Li}^{2} \cdot$ Yuhong Wang $^{5} \cdot$ Tao Chen $^{6}$. \\ Shaodong Hong ${ }^{2}$ (D) Li Zhang ${ }^{2}$
}

Published online: 6 December 2021

(c) Springer-Verlag GmbH Germany, part of Springer Nature 2021

Correction to: Cancer Immunology, Immunotherapy https://doi.org/10.1007/s00262-021-03082-4

The original version of this article unfortunately contained a mistake. The following article note should be included.

Zuan Lin, Xuanye Zhang and Yixin Zhou have contributed equally.

The original article has been corrected.

Publisher's Note Springer Nature remains neutral with regard to jurisdictional claims in published maps and institutional affiliations.

The original article can be found online at https://doi.org/10.1007/ s00262-021-03082-4.

Shaodong Hong

hongshd@sysucc.org.cn

$\triangle$ Li Zhang

Zhangli6@mail.sysu.edu.cn

1 Department of Clinical Research, Sun Yat-Sen University Cancer Center, Guangzhou, China

2 Department of Medical Oncology, Sun Yat-Sen University Cancer Center, Guangzhou, China

3 Department of VIP Region, Sun Yat-Sen University Cancer Center, Guangzhou, China

4 Department of Radiotherapy, Sun Yat-Sen University Cancer Center, Guangzhou, China

5 Department of Endoscopy, Sun Yat-Sen University Cancer Center, Guangzhou, China

6 Department of Nuclear Medicine, Sun Yat-Sen University Cancer Center, Guangzhou, China 